\title{
Non-Surgical Treatment with Chemical Peeling and Subsequent Vitamin C Iontophoresis for Rolling Scars in Acne Vulgaris
}

\author{
Ichiro Kurokawa \\ Department of Dermatology, Meiwa Hospital, Hyogo, Japan \\ Email: kurokawa.i@meiwa-hospital.com
}

How to cite this paper: Kurokawa, I. (2020) Non-Surgical Treatment with Chemical Peeling and Subsequent Vitamin C Iontophoresis for Rolling Scars in Acne Vulgaris. Journal of Cosmetics, Dermatological Sciences and Applications, 10, 104-106. https://doi.org/10.4236/jcdsa.2020.103011

Received: July 11, 2020

Accepted: August 23, 2020

Published: August 26, 2020

Copyright $\odot 2020$ by author(s) and Scientific Research Publishing Inc. This work is licensed under the Creative Commons Attribution International License (CC BY 4.0).

http://creativecommons.org/licenses/by/4.0/

\begin{abstract}
A 19-year-old man had atrophic scars on bilateral temple for several years. The atrophic scars were composed of rolling scars (three depressions, $8 \times 4$ $\mathrm{mm}$ in diameter), ice pick scars and boxcar scar. The patient was treated with chemical peeling (CP) using $20 \%$ glycolic acid (GA) (pH 3.2) and subsequent iontophoresis with vitamin $\mathrm{C}$ derivative at one month's interval twice. Remarkable improvement was observed; the rolling scars almost disappeared with traces of hypopigmented macule. However, the ice pick scars and boxcar scar did not improve. $\mathrm{CP}$ and subsequent vitamin $\mathrm{C}$ iontophoresis can be an alternative non-surgical and non-invasive treatment for rolling scar in acne.
\end{abstract}

\section{Keywords}

Atrophic Scars, Chemical Peeling, Non-Surgical Treatment

\section{Case Report}

A 19-year-old man had atrophic scars on bilateral temple for several years. The atrophic scars were composed of rolling scars (three depressions, $8 \times 4 \mathrm{~mm}$ in diameter), ice pick scars and boxcar scar (Figure 1(a)). The hypopigmented depressions of the rolling scars had a resulting undulating appearance and broadly based skin surface with poorly demarcated border (Figure 1(a)). The patient was treated with chemical peeling (CP) using $20 \%$ glycolic acid (GA) ( $\mathrm{pH}$ 3.2) and subsequent iontophoresis with Moisture Gel (Environ; protea Japan, Tokyo, Japan) at one month's interval twice. Moisture Gel contains vitamin C (ascorbic acid), vitamin $\mathrm{A}$ (retinyl palmitate), vitamin $\mathrm{E}$, vitamin $\mathrm{B} 5$ and $\beta$-carotene.

Remarkable improvement was observed; the rolling scars almost disappeared 


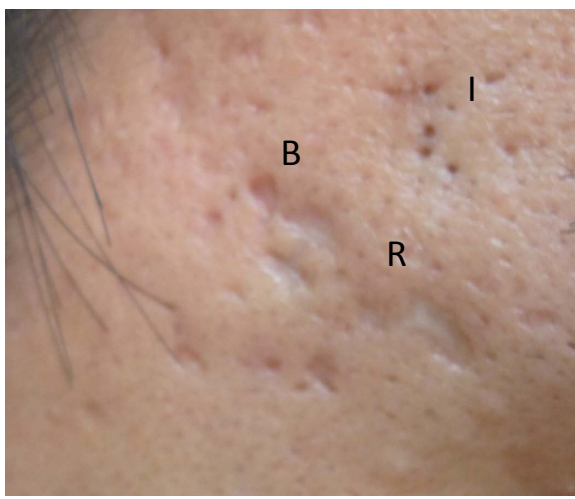

(a)

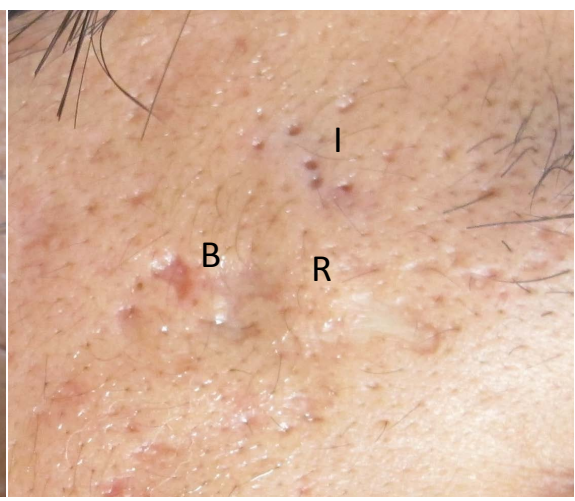

(b)

Figure 1. (a) Before the treatment, rolling scars (R), ice pick scars (I) and boxcar scar (B) were observed on the temple. The surface of rolling scars (R) are hypopigmented depressed and undulating with broad based skin surface; (b) After the treatment of chemical peeling by $20 \% \mathrm{GA}(\mathrm{pH} 3.2)$ and subsequent vitamin $\mathrm{C}$ iontophoresis twice at one month's interval, remarkable improvement was observed in the rolling scars (R). The rolling scars almost disappeared with trace hypopigmented macule. However, ice pick scars (I) and boxcar scar (B) did not improve.

with traces of hypopigmented macule (Figure 1(b)). However, the ice pick scars and boxcar scar did not improve.

Atrophic scar is one of the most important complications in acne vulgaris. Jacob et al. classified three types of atrophic scars in acne: icepick scar, rolling scar and boxcar scar [1]. Icepick scar is narrow $(<2 \mathrm{~mm})$ (V-shaped) sharply delineated and extended and tapered into the deep dermis and subcutis. Rolling scar is wider than 4 to $5 \mathrm{~mm}$ and has broad based skin surface with poorly demarcated depression and resultant undulating appearance (M-shaped). It is very superficial with a vertical extension that is limited to a depth corresponding to the epidermal thickness. Boxcar scar is round oval shaped and punch out with broad relatively flat base with vertical edge (U-shaped). However, these three types of scars are not always distinguishable.

Recent treatments for atrophic scar are laser resurfacing, emerging laser technologies, dermabrasion, chemical peeling, chemical reconstruction of skin scars (CROSS) technique, micro-needling, radiofrequency, fillers, punch excision and punch elevation, subcision and autologous platelet-rich plasma [2].

The pathogenesis of atrophic scar formation is unclear. The mechanism of rolling scar formation is believed to involve dermal tethering of the dermis to the subcutis. Abnormal fibrous anchoring of the dermis to the subcutis leads to superficial shadowing and rolling or undulating appearance to the overlying skin [3]. Therefore, rolling scar is preferentially treated by subcision to loosen the force of fiber in the dermis [1]. In addition, surgical treatments such as micro-needling and dermal fillers are performed [4], and most recent thread-lift technique [5]. CP is a novel non-surgical treatment for acne scar [6]. In particular, CP by GA is a non-invasive and non-harmful treatment. $\mathrm{CP}$ is believed to be effective for macular scar such as postinflammatory hyperpigmentation (PIH). 
However, icepick scar and rolling scar do not improve following CP according to the previous reports [3]. CP can regenerate the renewal of basal cells in the epidermis, enable skin remodeling, promote turnover of keratinocytes, proliferate fibroblasts and regulate cytokine network. Mild erythema and irritation are side effects of GA. If the epidermis remained at base of scar such as superficial rolling scar, the keratinocytes in the epidermis can proliferate, resulting in re-epithelialization and suppling the depressed defect of epidermis. In addition, vitamin $\mathrm{C}$ can also promote wound healing [7] in this case. Recent study [8] showed altered metabolism of elastic fibers and collagen fibers on transforming growth factor- $\beta 1$ signalling is involved in formation of atrophic scar in acne. As the number of this study is small, the limitation still remains.

$\mathrm{CP}$ and subsequent vitamin $\mathrm{C}$ iontophoresis can be an alternative non-surgical and non-invasive treatment for rolling scar in acne.

\section{Conflicts of Interest}

The author declares no conflicts of interest regarding the publication of this paper.

\section{References}

[1] Jacob, C.I., Dover, J.S. and Kaminer, M.S. (2001) Acne Scarring: A Classification System and Review of Treatment Options. Journal of the American Academy of Dermatology, 45, 109-117. https://doi.org/10.1067/mjd.2001.113451

[2] Connolly, D., Vu, H.L., Mariwalla, K. and Saedi, N. (2017) Acne Scarring-Pathogeneisis, Evaluation, and Treatment Options. Journal of Clinical and Aesthetic Dermatology, 10, 12-23.

[3] Fabbrocini, G., Annunziata, M.C., D’Arco, V., et al. (2010) Acne Scars: Pathogenesis, Classification and Treatment. Dermatology Research and Practice, 2010, Article ID: 893080. https://doi.org/10.1155/2010/893080

[4] Kravvas, G. and Al-Niaimi, F. (2017) A Systematic Review of Treatments for Acne Scarring. Part 1: Non-Energy-Based Techniques. Scars, Burns \& Healing, 3, 1-17. https://doi.org/10.1177/2059513117695312

[5] Donnarumma, M., Vastarella, M., Ferrillo, M., Cantelli, M., D’andrea, M. and Fabbrocini, G. (2019) An Innovative Treatment for Acne Scars With Thread-Lift Technique: Our Experience. Giornale Italiano di Dermatologia e Venereologia, Online Ahead of Print.

[6] Committee for Guidelines of Care for Chemical Peeling (2012) Guidelines for Chemical Peeling in Japan (3rd Edition). Journal of Dermatology, 39, 321-325.

https://doi.org/10.1111/j.1346-8138.2011.01362.x

[7] Kurokawa, I., Yoshioka, M. and Ito, S. (2019) Split-face Comparative Clinical Trial Using Glyceryl-Octyl-Ascorbic Acid/Ascorbyl 2-Phosphate 6-Palmitate/DL-a-tocopherol Phosphate Complex Treatment for Postinflammatory Hyperpigmentation, Postinflammatory Erythema and Atrophic Scar in Acne Vulgaris. Journal of Dermatology, 46, e347-e348. https://doi.org/10.1111/1346-8138.14930

[8] Moon, J., Yoon, J.Y., Yang, J.H., Kwon, H.H., Min, S. and Suh, D.H. (2019) Atrophic Acne Scar: A Process from Altered Metabolism of Elastic Fibres and Collagen Fibres Based on Transforming Growth Factor- $\beta 1$ Signalling. British Journal of Dermatology, 181, 1226-1237. https://doi.org/10.1111/bjd.17851 